\title{
Design of Security Monitoring System for Smart Home System Under the Background of Internet of Things Technology
}

\author{
Xuelei Fu \\ Guangdong Preschool Normal College in Maoming, Maoming, Guangdong, 525000, China \\ leigefu@163.com
}

Keywords: Smart Home System, Security Monitoring System, Internet of Things Technology.

\begin{abstract}
With the continuous development of Internet of things technology, the information exchange between "things" and people ," things "and" things "is realized, and the scope of information sharing is expanded, which provides technical guarantee for the monitoring and design of smart home system and meets the needs of people's home security. Based on this, the article will analyze the security monitoring design of smart home system from many aspects, in order to promote the development of smart home research system in China.
\end{abstract}

\section{Overview of Smart Home Systems}

With the acceleration of the process of urban construction, a large number of people pour into the city, giving new impetus to urban development, but also caused the problem of urban security, and family security has been paid more and more attention. In addition, a growing number of families, working hours at home no one, once there is a fire, leakage of water pipes will cause huge economic losses. Based on the background of Internet of things technology, integrating smart home into home security system can improve the level of home security. As the core part of the smart home system, the security monitoring system can monitor the state of the home in all directions, not only to let the user know the use of the home equipment remotely, but also to play the role of home theft prevention.

\subsection{Definition}

Smart home, also known as smart home, refers to the use of Internet of things technology to achieve intelligent home control (figure 1: smart home). Internet of things technology applied to smart home mainly includes: (1) intelligent remote control ;(2) computer technology ;(3) network communication technology; (4) integrated wiring technology ;(5) ergonomics[1]. Digital Home, Internet Home, Digital Home and Smart Home have similar meanings.

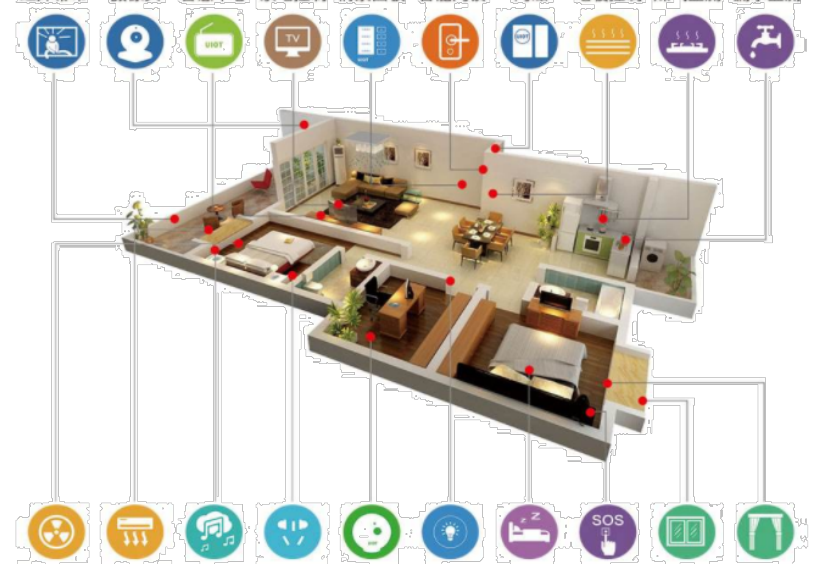

Figure 1 Smart home

Smart home has many subsystems :(1) light control system ;(2) gas valve control system ;(3) scene linkage;(4) floor heating ;(5) security system and other aspects. The security monitoring system is applied to every subsystem of smart home. It can obtain home information through 
sensors. It is the basic content of smart home system. The main features are as follows:

\subsection{Reliability}

The smart home system should have the reliability characteristic, can provide the service to the user all the time, avoids because the system malfunction, the blackout accident, the external force destruction causes the smart home to stop working, causes the influence to the user's life.

\subsection{Standards}

Smart home systems should have standard features, be compatible with a variety of smart home products at the same time, ensure system scalability, and allow different manufacturers to interconnect and share smart home products. Therefore, when setting up the front end of the smart home system, we should adopt the standardized interface design, let the smart home system integrate the platform, and have the characteristics of expansion, which can add the platform function by adding different factory equipment products.

\subsection{Convenience}

Smart home has the characteristics of convenience, this convenience is reflected in the construction and use of two aspects:(1) construction: wiring installation is relatively simple, and can be expanded according to the actual situation ;(2) use: smart home system is very convenient to use, users can achieve home intelligent control through intelligent control system.

\subsection{Development}

The real sense of smart home emerged in 1984, when the United States built the world's first smart building. With the concept of smart home put forward immediately popular, Europe, America, Japan and other developed countries have carried out in-depth research on this, and continue to develop smart home.The essence of smart home is to realize the control of "things" in the home by means of information technology, so the security monitoring system is the key technology to realize the management of smart home.

\subsection{Standards}

Smart Home Design Authority Standard:

Home Automation Systems and Communication Standards (1988): This standard, promulgated by the American Association of Electronic Industries, is groundbreaking for smart home development.

Guidelines for Electrical Design (Standards) of Well-off Residential Buildings (1997): In this discussion paper, it is proposed that China's smart home should meet five conditions :(1) comfortable living environment;(2) comprehensive information service capability ;(3) convenient communication mode ;(4) home intelligent system ;(5) high security[1].

\section{Overview of Security Monitoring System}

\subsection{Definition}

The security monitoring system refers to the system that monitors the activities of objects through the technology of host activity, real-time monitoring network and so on[2](Figure 2: Family Safety Monitoring System).The security monitoring system can sensitively evaluate the abnormal behavior and data in the system, and can track the data of the target object and record the relevant logs. 


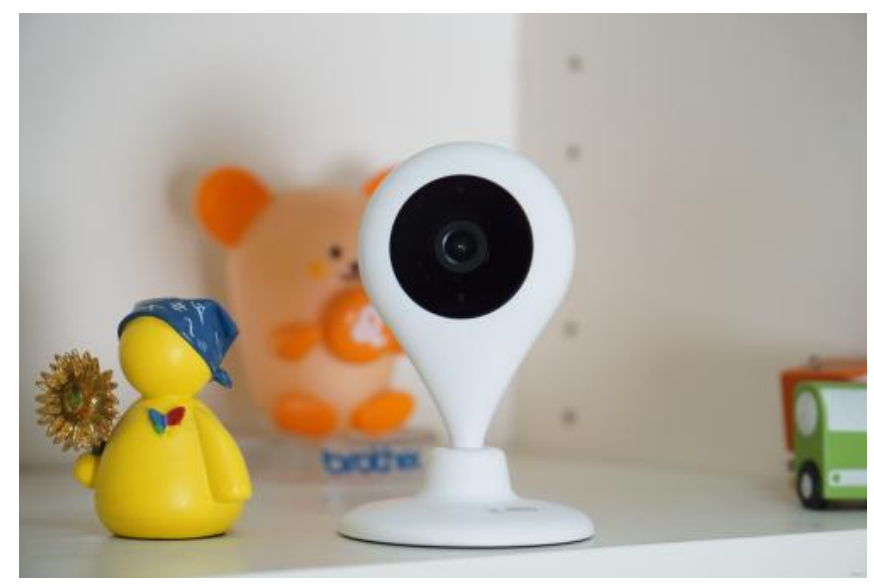

Figure 2 Family security monitoring system

\subsection{Smart Home Security Monitoring System}

Smart home security monitoring system is the basic guarantee to realize all functions of smart home. The "object" in the smart home system has the function of identifying, storing information, transmitting information to the system through the designated channel, and executing the commands issued in the system. In this process, the security monitoring system plays a great role, providing the data base for the smart home system through sensors (figure 3: infrared detector), remote monitoring and other technologies, and realizing remote monitoring.

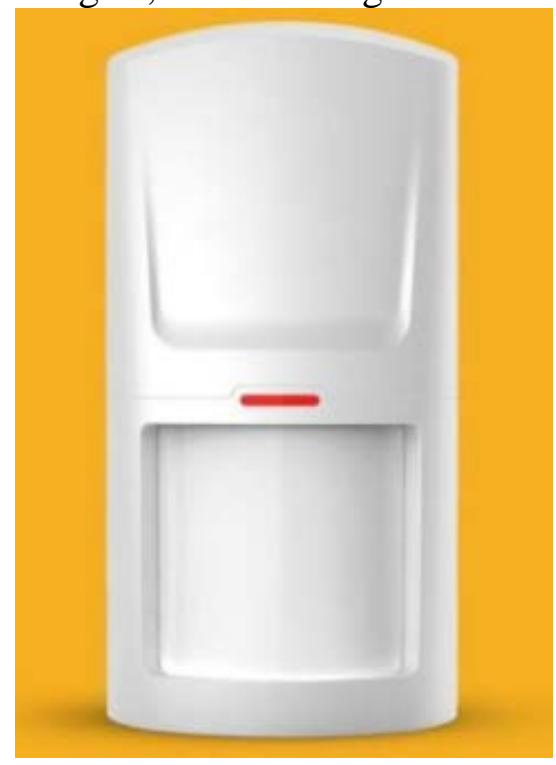

Figure 3 Infrared detectors

\section{Function Analysis of Smart Home Security Monitoring System}

The smart home system supported by the Internet of things technology is to apply the ability of "things" and people ," things "and" things "in the Internet of things to communicate, communicate and share information in the smart home system, so that users can obtain home information remotely and realize collectivization and convenient control. From the actual effect, the smart home system transforms the "real scene" control into virtual scene control, and builds an integrated intelligent system. According to the common design structure of modern smart home safety monitoring, it is mainly divided into four parts:

(1) Home environment monitoring: the main objects of safety environment monitoring are air temperature, humidity, light condition and CO.2content, the sensor is able to obtain the data of various indices in the air through external perception, and transmit it to the central control system to control the data values. In the residential environment monitoring, some home systems carry more 
advanced technology, can achieve window opening and other functions.

(2) Security control: including fire (temperature detection, gas detection), theft (image recognition), gas or toxic gas (gas detection), etc. Security monitoring and control system can detect temperature, humidity, gas, personnel intrusion, and timely alarm through the remote monitoring system, not only can notify users, but also can be linked to the police station, residential supervision office.

(3) Home appliance control: the smart home integrates all the smart home appliances into the system, integrates all the information, installs the WSN stage on the control equipment, and through the RS485 series all the home appliances, lets each node form the network, satisfies the system center control demand. The central control system can also control all household appliances through this path.For example: the user in the remote monitoring process, found that the bedroom lights on, you can control the lights off by remote.

(4) Video surveillance: Video surveillance mainly monitors the internal audio and video of the home through surveillance equipment to obtain all kinds of data during the operation of the equipment. Video surveillance can be stored in the internal settings of memory, can also be uploaded to the platform cloud disk system, users can view real-time and after-view surveillance video, to meet the needs of home security.

\section{Structure Design of Smart Home Security Monitoring System}

Smart home security monitoring system should include four parts:

First, WSN terminal acquisition control. By means of system sensors and WSN terminal nodes to collect environmental data and upload terminal data to the control system, users can control all home.

Second, WSN data base station control. The data function control effect of base station control part is more obvious, and the USB interface, remote gateway (M2M) and so on are mainly used in the process of controlling data.[3]WSN the data base station control center platform does not have the special request to the computer, generally windows the system computer may satisfy the construction demand, as long as adds the control software to the computer, may realize the data storage, the transmission and the analysis function.

Third, remote login control system. During operation, the system mainly adopts B/S framework, which provides login and browsing framework for remote control of smart home security monitoring system.

Fourth, M2M remote gateway. When users use smart home system, they can not control the monitoring distance unchanged, but they can use M2M remote gateway to let users control the device remotely through mobile computer, mobile phone, IPAD.

\section{Hardware Design of Smart Home Security Monitoring System}

CC2430 chip (TI company) can be used in the system design process to make integrated highspeed microcontroller, mainly used to monitor video transceiver, instruction transmission. In the system hardware module design mainly includes the sensor and the gateway, collects the data of the monitored object in the system. In the process of system application, the sensor is mainly responsible for obtaining information, while the control center chip is responsible for transforming and processing data, and sending relevant information to the gateway.

At present, the existing platform has been able to carry more than a dozen sensors, and achieve a variety of data conversion, such as light recognition, pressure perception, noise management, temperature and humidity perception. Currently, sensors produced in the market can be mounted in ATOS systems. For example, in the illumination system, it is mainly composed of power module, light sensor, processing module and communication module. In this system, the power module is responsible for providing power energy; the data is obtained by the attention brightness sensor, and the data information is processed by the processing module, and finally the data information is transmitted through the communication module. 


\section{Conclusion}

The development of Internet of things technology has realized the information exchange of different forms of objects, provided new ideas for human information management, and promoted the development of smart home. As the core system of smart home, security monitoring system is the basis of information collection and remote monitoring. In the design of modern smart home security monitoring system, we should pay attention to the needs of users, build information platform through the application of sensors, cameras, electronic computers and other electronic devices, let users remote control home, and obtain home information to meet the needs of user home security and smart home use.

\section{Acknowledgements}

2018 Guangdong Science and Technology Innovation Strategy Special Fund Project "Research and Application of Smart Home Security Technology Based on Internet of Things and Cloud Computing" (2018S001411)

\section{References}

[1] Yang, Li., Feng, Juan. Lu, Xiuli., et al. Design of Intelligent Home Security Monitoring System Based on Internet of things. Modern Electronic Technology , vol. 42, no. 8, pp. 55-58, 2019.

[2] Donglin Li, Gurimira Kizilbek. Summary of Research on Smart Home System Based on ZigBee. Computer Age, no. 6, pp. 23-25, 2019.

[3] Li, Jinke. Design and Application of Intelligent Residential Security System under the Application of Internet of things Technology, Digital Users, vol. 24, no. 46, pp. 88, 2018. 